\title{
Article
}

\section{Perceptions of physiotherapists towards research: a mixed methods study}

Janssen, J., Hale, L., Mirfin-Veitch, B. and Harland, T.

Available at http://clok.uclan.ac.uk/13735/

Janssen, J. ORCID: 0000-0002-5961-2736, Hale, L., Mirfin-Veitch, B. and Harland, T. (2016) Perceptions of physiotherapists towards research: a mixed methods study. Physiotherapy, 102 (2). pp. 210-216. ISSN 00319406

It is advisable to refer to the publisher's version if you intend to cite from the work. http://dx.doi.org/10.1016/j.physio.2015.04.007

For more information about UCLan's research in this area go to

http://www.uclan.ac.uk/researchgroups/ and search for < name of research Group>.

For information about Research generally at UCLan please go to http://www.uclan.ac.uk/research/

All outputs in CLoK are protected by Intellectual Property Rights law, including Copyright law. Copyright, IPR and Moral Rights for the works on this site are retained by the individual authors and/or other copyright owners. Terms and conditions for use of this material are defined in the policies page.

\section{CLoK}

Central Lancashire online Knowledge www.clok.uclan.ac.uk

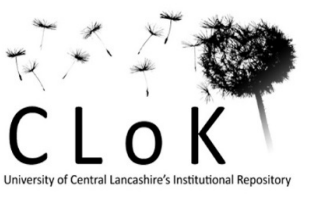


Perceptions of physiotherapists towards research: a mixed methods study

J Janssen, L. Hale, B. Mirfin-Veitch, T. Harland

Author accepted manuscript

Online 17 June 2015

Physiotherapy 2016; in press

doi:10.1016/j.physio.2015.04.007 


\section{Abstract \\ Objectives}

To explore the perceptions of physiotherapists towards the use of and participation in research.

Design

Concurrent mixed methods research, combining in-depth interviews with three questionnaires (demographics, Edmonton Research Orientation Survey, visual analogue scales for confidence and motivation to participate in research).

\section{Setting}

One physiotherapy department in a rehabilitation hospital, consisting of seven specialised areas.

\section{Participants}

Twenty-five subjects \{four men and 21 women, mean age 38 [standard deviation (SD) 11] years \} who had been registered as a physiotherapist for a mean period of 15 (SD 10) years participated in this study. They were registered with the New Zealand Board of Physiotherapy, held a current practising certificate, and were working as a physiotherapist or physiotherapy/allied health manager at the hospital.

\section{Main outcome measure}

The primary outcome measure was in-depth interviews and the secondary outcome measures were the three questionnaires.

Results

Physiotherapists were generally positive towards research, but struggled with the concept of research, the available literature and the time to commit to research. Individual confidence and orientation towards research seemed to influence how these barriers were perceived.

\section{Conclusion}

This study showed that physiotherapists struggle to implement research in their daily practice and become involved in research. Changing physiotherapists' conceptions of research, making it more accessible and providing dedicated research time could facilitate increased involvement in the physiotherapy profession.

Keywords

Physiotherapy. Research perception, Research utilisation, Research participation, Practiceresearch gap, Evidence-based practice 
Introduction

Evidence-based practice plays an important role in the current physiotherapy profession [1]. The research from which evidence is derived is translated into daily practice but only a limited number of physiotherapists are involved in the research process itself [2]. Therefore they utilise rather than create knowledge. Since the 1980's the factors that influence research use have been investigated [3]. Most studies focus on the individual attributes of clinicians. For example, a systematic review in 2003 of the nursing literature established a positive link between attitude towards research and the use of research [4]. In allied health research, therapists held a more positive attitude towards research use than nurses [5] and generally believed it is important for their profession [6-10]. Allied health research also found that both attitudes [11] and research skills [8, 10-14] influence the use of research in practice. In addition, the culture in which the clinician operates is known to play an important role in the uptake of research [15-19]. For example, a survey-based study of 458 nurses revealed that a lack of autonomy created an unsupportive environment for using research [19]. The Promoting Action on Research Implementation in Health Services (PARIHS) model identified that higher levels of research use were found in hospitals that encouraged staff to keep on learning and developing, and that had supportive leadership $[15,16]$. Other barriers relate to research characteristics, such as being able to analyse statistics, understand limited or conflicting results, not being able to access the evidence-base and difficulty translating results into practice $[3,6,10,13,20]$.

Providing physiotherapists with the opportunity to conduct research could be a way to build evidence that can be used to inform the physiotherapy profession, change attitudes towards research and develop research skills [10]. However, the perceptions that physiotherapists hold about participating in their own research are currently unknown. In the present study we explored what physiotherapists perceived as the barriers to using and participating in research. 
The work is part of a larger study that aimed to stimulate clinical physiotherapists to conduct research in their own setting [21].

Methods

\section{Setting of the study}

This study was carried out in New Zealand, where the evidence based practice movement plays a major role in the practice of physiotherapy [22]. The Health Practitioners Competence Assurance Act [23] stipulates that every physiotherapist in New Zealand registers with the Physiotherapy Board of New Zealand and annually has to apply for a practising certificate. The Physiotherapy Board of New Zealand requires physiotherapists to implement evidence of learning into their practice; to be up to date with current research, and to evaluate their practice with audits and self-reflection [24]. In addition the Accident Compensation Corporation (ACC) [25], a governmental organisation that funds physiotherapy sessions for people with injuries resulting from an accident, requires physiotherapists to implement evidence in their practices and reporting to ACC. Consequences of not meeting the stipulated requirements could result in restricted funding for the treatment of that specific patient [26].

The setting was a New Zealand rehabilitation hospital that encompassed a range of clinical specialties, including in- and outpatient settings. Some of these specialities were already involved in research prior to the start of this study, whereas most of the others were not. The two clinical managers each had their own responsibility for a number of specialities; one of these managers was in favour of research, while the other was not. Each physiotherapist was allocated a yearly allowance of CPD activities, also monthly CPD sessions took place for the 
entire physiotherapy department and knowledge gained from courses or conferences was shared.

\section{$\underline{\text { Design }}$}

The study incorporated a concurrent mixed-method research design [27] to collect qualitative (semi-structured interviews) and quantitative (three questionnaires) data. Mixed-method research enables both qualitative and quantitative data to be used to answer the research question [27]. A pragmatic paradigm [28] underpinned the study as it answered the research question. Furthermore, the practical background of the first author (physiotherapist and applied researcher) suited this paradigm. Priority has been given to the qualitative data as it provided for a more in-depth analysis of the perceptions of the individual participants. The quantitative date was then used to further inform the findings of the qualitative analysis. The (name) Upper South A Regional Ethics Committee approved this study.

Participant recruitment

All physiotherapists and clinical managers working in the Physiotherapy department of the rehabilitation hospital were invited to participate. Volunteers were included when they were: employed as either a physiotherapist, or a Physiotherapy or allied health manager at the hospital; registered with the New Zealand Board of Physiotherapy; holders of a Current Practicing Certificate. Information sheets were sent to all potential participants and two oral presentations were held to explain the study. Twenty five volunteers provided written consent in the two weeks after the last presentation and were included in the study.

\section{$\underline{\text { Data collection }}$}

Qualitative and quantitative data were collected. After consent was obtained, the quantitative questionnaires were sent to all participants. Following completion of the questionnaires, an individual qualitative interview took place. In-depth face-to-face individual interviews formed 
the main data source. The interview schedule is presented in Table 1. The questions were semistructured and allowed for exploratory questions. Physiotherapists and their managers were purposefully sampled to capture the maximum variation [29] in confidence and motivation towards research. The interviews took place prior to a participatory action research intervention that was designed to stimulate research participation [21]. Each interview was held in a private room within the hospital to create a space of reflection and trust. Interviews lasted from 20 to 60 minutes, were audio-recorded and transcribed verbatim.

The quantitative data were collected through three questionnaires. The first questionnaire collected demographic data, such as age, sex and number of years of Physiotherapy registration. The second questionnaire was the Edmonton Research Orientation Survey (EROS) [30]. The EROS consisted of three parts answered on a five-point Likert scale, ranging from strongly disagree (1) to strongly agree (5); a higher score indicating a more positive orientation towards research. Internal reliability was measured high (Cronbach's alpha $=0.93$ ) [30] and construct validity found the overall EROS score to be associated with formal research education, understanding of research, and participation in research amongst nurses [31]. The third questionnaire consisted of two visual analogue scales to measure motivation and confidence in conducting research. The two questions asked were: 1) How confident are you with doing research? 2) How motivated are you to do research? Answers were registered on a $100 \mathrm{~mm}$ visual analogue scale ranging from, respectively, not confident and not motivated to very confident and very motivated. Reliability and validity of this last questionnaire has not been established.

\section{$\underline{\text { Data analysis }}$}

Qualitative and quantitative data were analysed separately and later combined. The in-depth interviews were transcribed verbatim, checked for accuracy by one researcher and authenticity 
by the participants. After the transcripts were anonymised they were imported into NVivo8 software package (QSR international). The transcripts were thematically analysed according to the guidelines of Braun and Clarke [32]. This study focused on the perceptions of the physiotherapists, therefore interview data were analysed first. The first author read and listened to all recorded qualitative data sources. All transcripts were coded by the first author, but all authors discussed and verified emerging themes. Fifteen physiotherapists and/or managers were interviewed at which point data saturation was reached.

Quantitative data are presented as means and standard deviation. Due to the small sample size of this study, further analysis was not appropriate.

Results

Twenty five physiotherapists, 21 females and 4 males (mean age 38 years, SD 11 years) registered as a physiotherapist for a mean period of 15 years (SD 10 years) were included. Confidence in conducting research ranged from 0 to 100 (mean confidence 38, SD 27) and motivation to participate in a research project ranged from 18 to100 (mean motivation 63, SD 25). More information is displayed in Table 2. Despite the fact that $56 \%$ had not attended a research course and $60 \%$ did not spend time on research activities, $44 \%$ of the physiotherapists read articles in their own time.

The fifteen physiotherapists who were interviewed consisted of 12 females and 3 males. On average they were 42 years old (SD 11 years) and had been registered as a physiotherapist for 17 years (SD 11 years). The range in confidence and motivation to conduct research was similar to the overall group.

Three themes emerged from the analysis: 1) the concept of research, 2) the available research and 3) time and leadership. Overall physiotherapists saw research as something very positive 
for their profession. A commonly held view was that research had shaped the physiotherapy profession to what it is now, and also increased the professional status of the physiotherapy profession. One of the physiotherapists expressed these ideas through the following comment:

You know, we can't rely on 'Oh well, they said they got better.' We need to have evidence to really highlight those changes and yeah, I think that research buys us that academic title and also it should cement our position within the healthcare system as well.

(Physiotherapist 13, confidence 58, motivation 78, EROS 3.92)

Not all physiotherapists shared these views. Two expressed concerns about evidence based practice and the implications it could have for therapists and patients:

We're just losing our profession in that respect because we're now legally bound to try and work within evidence based practice and there's no such real thing. In the majority of our physiotherapy, realistically ... you know, you can't measure it, you can't research it. The only way you can do it is to look at the marketplace. And if we're not offering it, that patient, if they have the money, will go elsewhere to find it.

(Physiotherapist 15, confidence 7, motivation 63, EROS 3.11)

\section{Theme 1: The concept of research}

This theme encompassed the notion of how research was viewed. Physiotherapists wanted research to answer the questions in order to enhance practice. The majority saw it as something very complicated and academic, and that research needed a very specific focus:

Well I guess my concept is that it's quite academic. There'd be a lot of work involved. ... you actually have to do something quite specific in the end because if it's too 
broad, it's just too big and you can't do it.

(Physiotherapist 21, confidence19, motivation 48, EROS 2.84)

Regarding research type, randomised controlled trials (RCTs) and systematic reviews were mentioned as being the main source of information. Case studies or case series were seen as less scientific as it was difficult to generalize the findings. Qualitative research was not mentioned. Those who were interested in joining the participatory action research intervention mainly talked about doing a RCT and were reluctant to investigate their practice on a smaller scale. These views were held despite the fact that the majority of participants were involved in audits or service evaluations within their own areas. Research was seen as a large published study.

\section{Theme 2: The available research}

The amount, language used and quality of the available literature influenced the perception of physiotherapists towards research in multiple ways. Physiotherapists who were not confident to participate in research also struggled to access the electronic databases they required.

I look things up but you know, I'm not sure what I'm looking at, to be honest. I will look things up, you know, and type in a few key words.

(Physiotherapist 19 confidence 12, motivation 27, EROS 2.95) Other physiotherapists who were assessed as having high levels of research confidence found the amount of literature available to be overwhelming.

You see an [interesting] journal and there's 12 articles and you can see a relationship with your work in probably ten of them .... so you're left with probably a week's worth of reading on top of everything else. I've got information overload. So that's 
probably a barrier, the sheer volume.

(Physiotherapist 3, confidence 54, motivation 86, EROS 4.03)

Such statements were generally followed by a remark about the language used in academic articles or the quality of these. Some found it difficult to understand what the researchers had written, while others found it difficult to assess the quality of the findings:

I mean some papers you just can't read [ ] All the statistics, and you're saying, 'What is this actually saying to me?' So you just read the conclusion or the summary.

(Physiotherapist 15, confidence 7, motivation 63, EROS 3.11) How to tell a good article from not such a good article? I really haven't got too much idea about how you assess that.

(Physiotherapist 21, confidence19, motivation 48, EROS 2.84)

Interestingly the majority of the physiotherapists interpreted the limitation section and the sentence 'more research is required' as a sign of weakness in a paper and felt frustrated that after all the effort they put in to read the paper, no definite conclusion could be drawn.

The majority of the papers I've read all say 'More research is required.' That they haven't actually come to a decent conclusion

(Physiotherapist 15, confidence 7, motivation 63, EROS 3.11)

\section{Time and leadership:}

Physiotherapists felt that due to the pressures of their practical work they did not have adequate time available to become involved in research. This barrier was mentioned by both full time and part time staff, and was independent of the orientation and confidence physiotherapists had towards research. 
Trying to get time, a substantial block of time without patient contact obviously is really difficult because you're carrying a full workload.

(Physiotherapist 23, confidence 100, motivation 100, EROS 3.84)

You know, I'm there for the patients really. So it's a fine balance, and especially as a part-timer, that fine balance is actually tipped very quickly.

(Physiotherapist 15, confidence 7, motivation 63, EROS 3.11)

Physiotherapists in senior positions and managers were seen as role models and their leadership affected how time was perceived.

Negotiation with management .... If management really valued research, then they would be making time within the clinicians' week, if not the clinicians' day, to engage in that research.

(Physiotherapist 3, confidence 54, motivation 86, EROS 4.03)

When managers or senior physiotherapists were involved in research, physiotherapists in the same area seemed to be more focussed towards research and were more flexible with their time.

I think there may have to be a bit of juggling with time and a few other responsibilities and things, but depending on how big the project was and how much you were involved in it, but I do think there is room for it.

(Physiotherapist 12, confidence 24, motivation 79, EROS 3.34)

\section{Discussion}

This research showed that the majority of this group of physiotherapists perceived research to be important for their profession. This is in line with previous studies $[6-8,10]$. However it is 
interesting to note that the EROS scores evident in this study still bordered on the lower margins of the EROS scores of allied health professions [2].

This study also presented three themes that influenced physiotherapists' use of and participation in research: the concept of research; the quality, language and amount of available research; and time and leadership available to use and participate in research. Even though the research question explored how physiotherapists perceived the barriers to use of research and participation in research, the majority offered their perceptions about utilisation but were less inclined to discuss issues related to participation. This resonates with findings of Stephens et al. and Kamwendo et al. who reported allied health professionals to be more interested in research use than in participation $[2,33]$.

The first theme, the concept of research, showed that the majority of those participants interviewed perceived good research to consist of randomised controlled trials and reviews. This implies that the physiotherapists' views were largely influenced by positivist views of knowledge through the evidence-based medicine paradigm, which ranks the evidence of a scientific publication [1]. Although this finding verified that this paradigm is accepted and used in the physiotherapy profession as a research utilisation tool, it could inhibit physiotherapists wanting to carry out research as systematic reviews and RCT's are generally not easy to do. Other types of research that were not considered, such as case studies and qualitative studies, should not be discarded as these provide an entry point to learning research in a time-restricted environment, and so a voice to clinical experiences [34].

The second theme related to the amount, language used and quality of the available literature. Attitude towards research and confidence in conducting research played a role in how 
physiotherapists perceived this barrier. The majority of participants felt their confidence to conduct research to be low and found it difficult to access databases. This difficulty corresponds with findings presented by Metcalfe et al [6] where the majority of the surveyed allied health professionals cited their low research skills as being one of the reasons that they did not use research in practice. However, physiotherapists with more confidence in research perceived the quantity of relevant literature to be overwhelming. In addition, researchconfident physiotherapists felt overwhelmed with the volume of new papers. However, the availability of allocated research time plays an important role in this finding $[6,8,10,13,18$, $20,35]$. The study showed that physiotherapists appeared to be discouraged by the frequently used conclusion that 'more research is required'. They interpreted this phrase as indicating low quality research, however, it is likely that they understood the value of such a conclusion while being reminded that good evidence that would directly inform their practice is a challenge to produce. In order to encourage physiotherapists' interest in participating in research, making the topic and study relevant for clinicians has proven to be a successful strategy [36].

The final theme concerned time and the role of leaders for the physiotherapist to use research. Time has long been recognised as the biggest barrier to reading and using research in practice $[6,8,10,13,20,35]$. Cultures within the hospital are likely to have played a big role in this finding $[16,32]$. In the nursing literature it has been established that culture can have a great impact on clinicians involvement in research, however nurses tend to have less autonomy than physiotherapists [19]. Rycoft-Malone highlighted that hospitals with a learning culture, in combination with leaders who facilitate learning, and who evaluate their own practices, should be more successful in implementing evidence-based practice in their daily work [17, 37]. In this study it has also been illustrated that physitoherapists who were supported by their 
manager or senior physiotherapist to do research, were more appreciative of research and found more time to be active in research.

Limitations of the current study include that although physiotherapists worked in different specialisations, they all worked at the same hospital. Furthermore a higher percentage of physiotherapists with Diplomas were interviewed compared to physiotherapists with a Bachelor degree or higher. This might have led to an under appreciation towards research as physiotherapists with Diplomas would not have been educated in research in their degrees. Characteristics of this hospital, in addition to cultural aspects of the country where this research was conducted may therefore have influenced the findings of this study.

\section{Conclusion}

This study showed that physiotherapists struggle with implementing research in practice and that there were barriers to becoming involved in research. Changing the concept of research, making research more accessible, allocating time to read and practice research, and promoting a culture for change could facilitate more involvement and enhance evidencebased practice. We suggest three strategies for research engagement: 1) educating clinicians about how to assess and critique research papers supported by professional development sessions in the clinical site; 2) promoting the use of different research paradigms (e.g. qualitative or case studies) and 3) providing specific allocated time for research.

\section{Acknowledgements}

We would like to thank the physiotherapists who participated in this project.

Ethical approval: The Upper South A Regional Ethics Committee, New Zealand provided ethical approval. 
Funding for this project was provided by Burwood Academy of Independent Living,

Christchurch, New Zealand and the University of Otago, Dunedin, New Zealand

Conflict of interest: the authors declare no conflict of interest 


\section{References}

1. Evidence-Based Medicine Working Group, Evidence-based medicine. A new approach to teaching the practice of medicine. JAMA, 1992. 268(17): p. 2420-5.

2. Stephens, D., N. Taylor, and S.G. Leggat, Research experience and research interests of allied health professionals. Journal of Allied Health, 2009. 38(4): p. e107-111.

3. Champion, V.L. and A. Leach, Variables related to research utilization in nursing: an empirical investigation. Journal of Advanced Nursing, 1989. 14(9): p. 705-710.

4. Estabrooks, C.A., et al., Individual determinants of research utilization: a systematic review. Journal of Advanced Nursing, 2003. 43(5): p. 506-20.

5. Eller, L.S., E. Kleber, and S.L. Wang, Research knowledge, attitudes and practices of health professionals. Nursing Outlook, 2003. 51(4): p. 165-170.

6. Metcalfe, C., et al., Barriers to implementing the evidence base in four NHS therapies: Dietitians, occupational therapists, physiotherapists, speech and language therapists. Physiotherapy, 2001. 87(8): p. 433-41.

7. Shakeshaft, A.M., A study of the attitudes and perceived barriers to undertaking clinical governance activities of dietitians in a Welsh National Health Service trust. Journal of Human Nutrition \& Dietetics, 2008. 21: p. 225-238.

8. Iles, R. and M. Davidson, Evidence based practice: a survey of physiotherapists' current practice. Physiotherapy Research International, 2006. 11: p. 93-103.

9. Ballin, A.J., et al., Research in physical therapy: philosophy, barriers to involvement, and use among California physical therapists. Physical Therapy, 1980. 60(7): p. 88895.

10. Scurlock-Evans, L., P. Upton, and D. Upton, Evidence-Based Practice in physiotherapy: a systematic review of barriers, enablers and interventions. Physiotherapy, 2014(0).

11. Bridges, P., L. Bierema, and T. Valentine, The propensity to adopt evidence-based practice among physical therapists. BMC Health Services Research, 2007. 7(1): p. 103.

12. Byham-Gray, L.D., et al., Evidence-based practice: what are dietitians' perceptions, attitudes, and knowledge? Journal of the American Dietetic Association, 2005. 105(10): p. 1574-81.

13. Grimmer-Somers, K., et al., Perspectives on research evidence and clinical practice: a survey of Australian physiotherapists. Physiotherapy Research International, 2007. 12(3): p. 147-61.

14. Pain, K., et al., Effects of profession and facility type on research utilization by rehabilitation professionals. Journal of Allied Health, 2004. 33(1): p. 3-9.

15. Cummings, G.G., et al., Influence of organizational characteristics and context on research utilization. Nursing Research, 2007. 56(4S): p. S24-39.

16. McCormack, B., et al., Getting evidence into practice: the meaning of 'context'. Journal of Advanced Nursing, 2002. 38(1): p. 94-104.

17. Rycroft-Malone, J., Evidence-informed practice: from individual to context. Journal of Nursing Management, 2008. 16(4): p. 404-408.

18. Brown, C.E., et al., Multi-institutional study of barriers to research utilisation and evidence-based practice among hospital nurses. Journal of Clinical Nursing, 2009. 19(13-14): p. 1944-1951.

19. Brown, C.E., et al., Nursing practice, knowledge, attitudes and perceived barriers to evidence-based practice at an academic medical center. Journal of Advanced Nursing, 2009. 65: p. 371-381.

20. Pennington, L., Attitudes to and use of research in speech and language therapy. British Journal of Therapy and Rehabilitation, 2001. 8(10): p. 375-9. 
21. Janssen, J., et al., Building the research capacity of clinical physical therapists using a participatory action research approach. Physical Therapy, 2013. 93(7): p. 923-34.

22. New Zealand Society of Physiotherapists. Rules book. Last amended May 2010. 2010; Available from: http://www.physiotherapy.org.nz/Folder?Action=View\%20File\&Folder_id=119\&File $=$ Rules\%20May\%202010.pdf.

23. Health Practitioners Competence Assurance Act 2003.

24. New Zealand Society of Physiotherapists. Standards of Physiotherapy. 2008 November 2010 [cited 2010 December 12 ]; 4th Edition:[Available from: http://www.physiotherapy.org.nz/Category?Action=View\&Category_id=300.

25. ACC. Welcome to ACC. n.d. 15 December 2008; Available from: http://www.acc.co.nz.

26. ACC. Physiotherapy services interim contract. 2009; Available from: http://www.acc.co.nz/PRD_EXT_CSMP/groups/external_providers/documents/guide/ prd_ctrb120281.pdf.

27. Creswell, J.W., Research design: qualitative, quantitative, and mixed methods approaches. 2009, Thousand Oaks, CA: Sage Publication.

28. Greene, J.C. and V.J. Caracelli, Defining and describing the paradigm issue in mixedmethod evaluation, in Advances in mixed-method evaluation :the challenges and benefits of integrating diverse paradigms, J.C. Greene and V.J. Caracelli, Editors. 1997, Jossey-Bass Publishers: San Francisco.

29. Patton, M.Q., Designing qualitative studies, in Qualitative research and evaluation methods, M.Q. Patton, Editor. 2002, Sage Publications: Thousand Oaks, California. p. 598.

30. Pain, K., P. Hagler, and S. Warren, Development of an instrument to evaluate the research orientation of clinical professionals. Canadian Journal of Rehabilitation, 1996. 9(2): p. 93-100.

31. McCleary, L. and G.T. Brown, Use of the Edmonton Research Orientation Scale with nurses. Journal of Nursing Measurement, 2002. 10(3): p. 263-275.

32. Braun, V. and V. Clarke, Using thematic analysis in psychology. Qualitative Research in Psychology, 2006. 3: p. 77-101.

33. Kamwendo, K., What do Swedish physiotherapists feel about research? A survey of perceptions, attitudes, intentions and engagement. Physiotherapy Research International, 2002. 7(1): p. 23-34.

34. Bithell, C., Evidence-based Physiotherapy: Some thoughts on 'best evidence'. Physiotherapy, 2000. 86(2): p. 58-59.

35. Closs, S.J. and B.J. Lewin, Perceived barriers to research utilization: a survey of four therapies. British Journal of Therapy and Rehabilitation, 1998. 5(3): p. 151-155.

36. Henderson, A., et al., The motivation of health professionals to explore research evidence in their practice: An intervention study. Journal of Clinical Nursing, 2006. 15(12): p. 1559-64.

37. Rycroft-Malone, J., The PARIHS framework -- a framework for guiding the implementation of evidence-based practice... Promoting Action on Research Implementation in Health Services. Journal of Nursing Care Quality, 2004. 19(4): p. 297-304. 
Table 1 Semi structured interview questions

1) What does physiotherapy mean to you?

2) What do you think of the role research has in physiotherapy practice?

3) Can you give me an example of when you have used research?

4) What would you like to learn about research?

5) What do you think is possible to learn about research in this setting?

Questions were followed with prompts such as:

"Can you tell me a little bit more that?"

"Can you describe an example of when that happened?"

"How did that make you feel?" 
Table 2: Overview questionnaires mean (sd) for the overall group $(\mathrm{n}=25)$ and interview sample $(n=15)$

\begin{tabular}{|c|c|c|}
\hline & Overall group & Interview sample \\
\hline \multicolumn{3}{|l|}{ Demographics } \\
\hline Age (years) & $38(11)$ & $42(11)$ \\
\hline Gender & $\begin{array}{c}21 \text { females }(84 \%) \\
4 \text { males }(16 \%)\end{array}$ & $\begin{array}{l}12 \text { females }(80 \%) \\
3 \text { males }(20 \%)\end{array}$ \\
\hline Registered as a physiotherapist (years) & $15(10)$ & $17(10)$ \\
\hline Research degree & $\begin{array}{l}\mathrm{n}=8 \text { Diploma }(32 \%) \\
\mathrm{n}=15 \text { Bachelor }(60 \%) \\
\mathrm{n}=2 \text { Masters }(8 \%)\end{array}$ & $\begin{array}{c}\mathrm{n}=6 \text { Diploma }(40 \%), \\
\mathrm{n}=8 \text { Bachelor }(53 \%) \\
\mathrm{n}=1 \text { Masters }(7 \%)\end{array}$ \\
\hline \multicolumn{3}{|l|}{ Visual Analogue Scales: range 0-100 } \\
\hline Motivation level & $62.6(25.1)$ & $65.9(25.6)$ \\
\hline Confidence level & $38.1(27.1)$ & $34.9(27.8)$ \\
\hline \multicolumn{3}{|c|}{ Edmonton Research Orientation Survey: range 1-5 } \\
\hline EROS total score & $3.4(0.5)$ & $3.4(0.4)$ \\
\hline Sub-scale 1: Valuing research & $3.8(0.6)$ & $3.7(0.6)$ \\
\hline Sub-scale 2: Involvement in research & $2.5(0.7)$ & $2.4(0.7)$ \\
\hline Sub-scale 3: Being at the leading edge & $3.9(0.5)$ & $3.8(0.6)$ \\
\hline Sub-scale 4: Evidence based practice & $3.5(0.5)$ & $3.5(0.5)$ \\
\hline
\end{tabular}

